\title{
Josephson (001) tilt grain boundary junctions of high temperature superconductors
}

\author{
Gerald B. Arnold ${ }^{1, *}$ and Richard A. Klemm ${ }^{2, \text { t }}$ \\ ${ }^{1}$ Department of Physics, University of Notre Dame, Notre Dame, IN \\ ${ }^{2}$ Department of Physics, Kansas State University, Manhattan, KS 66506
}

(Dated: July 20, 2018)

\begin{abstract}
We calculate the Josephson critical current $I_{c}$ across in-plane (001) tilt grain boundary junctions of high temperature superconductors. We solve for the electronic states corresponding to the electrondoped cuprates, two slightly different hole-doped cuprates, and an extremely underdoped holedoped cuprate in each half-space, and weakly connect the two half-spaces by either specular or random Josephson tunneling. We treat symmetric, straight, and fully asymmetric junctions with $s^{-}$, extended- $s$, or $d_{x^{2}-y^{2}}$-wave order parameters. For symmetric junctions with random grain boundary tunneling, our results are generally in agreement with the Sigrist-Rice form for ideal junctions that has been used to interpret "phase-sensitive" experiments consisting of such in-plane grain boundary junctions. For specular grain boundary tunneling across symmetric junctions, our results depend upon the Fermi surface topology, but are usually rather consistent with the random facet model of Tsuei et al. [Phys. Rev. Lett. 73, 593 (1994)]. Our results for asymmetric junctions of electrondoped cuprates are in agreement with the Sigrist-Rice form. However, our results for asymmetric junctions of hole-doped cuprates show that the details of the Fermi surface topology and of the tunneling processes are both very important, so that the "phase-sensitive" experiments based upon in-plane Josephson junctions are less definitive than has generally been thought.
\end{abstract}

PACS numbers: $74.20 . \mathrm{Rp}, 74.50 .+\mathrm{r}, 74.78 . \mathrm{Bz}$

\section{INTRODUCTION}

Because of its relation to the mechanism for superconductivity in the high temperature superconducting compounds (HTSC), there has long been a raging debate regarding their orbital symmetry of the superconducting order parameter (OP) 1.2.3.4 Only "phasesensitive" experiments involving Josephson tunneling can distinguish the OP from the non-superconducting pseudogap ${ }^{1.4 .5}$ Although the first in-plane phasesensitive experiment on $\mathrm{YBa}_{2} \mathrm{Cu}_{3} \mathrm{O}_{7-\delta}$ (YBCO) suggested a dominant $s$-wave $\mathrm{OP}, \underline{\underline{6}}$ experiments using tricrystal films of $\mathrm{YBCO}, \mathrm{Bi}_{2} \mathrm{Sr}_{2} \mathrm{CaCu}_{2} \mathrm{O}_{8+\delta}$ ( $\mathrm{Bi2212}$ ), and $\mathrm{Nd}_{1.85} \mathrm{Ce}_{0.15} \mathrm{CuO}_{4+\delta}(\mathrm{NCCO})$, and tetracrystal films of $\mathrm{YBCO}$ and $\mathrm{La}_{2-x} \mathrm{Ce}_{x} \mathrm{CuO}_{4-y}$ (LCCO) suggested that these materials had a dominant $d_{x^{2}-y^{2}}$-wave $\mathrm{OP}, 1,7.8,9,10,11.12$

Very different results were obtained for $c$-axis junctions. Low temperature $T$-axis Josephson junctions between $\mathrm{Pb}$ and $\mathrm{YBCO}, \mathrm{Bi} 2212$, and $\mathrm{NCCO}$ suggested varying amounts of an $s$-wave OP component 13.14 .15 .16 .17 More recently, three Bi2212 c-axis twist Josephson junction experiments showed that the OP has at least a substantial, and possibly a dominant $s$-wave component for $T$ up to the transition temperature $T_{c}, 4,18,19,20,21$ Here we search for a reason for these qualitative differences ${ }^{4}$

To date, most theoretical treatments of in-plane (001) tilt grain boundary (GB) junctions either used the Ginzburg-Landau (GL) approach ${ }^{22}$ or assumed a circular in-plane Fermi surface (FS) cross-section, and that the FS-restricted $d_{x^{2}-y^{2} \text {-wave OP } \propto \cos \left(2 \phi_{\boldsymbol{k}}\right), 23,24,25,26,27}$ Most of those treatments focussed upon the single quasiparticle density of states on the surface, rather than the critical current across the misalignment grain boundary junctions. The only previous treatment that included a tight-binding FS was that of Shirai et al., for which the FS consisted of very small pockets centered at the corners of the first Brillouin zone (BZ) 28 Their chosen FS was extremely different from any that have been extracted from angle-resolved photoemission spectroscopy (ARPES) experiments ${ }^{29}$ Shirai et al. studied both the single quasiparticle and supercurrent properties of GB junctions constructed from four specific surfaces 28 Here we explicitly take account of the tight-binding hole and electron-doped FS's observed using ARPES and of the surface boundary conditions (BC's) at the interfaces.

We find that a $d_{x^{2}-y^{2}}$-wave OP can be consistent with the tricrystal experiments for electron-doped cuprates, for which the two-dimensional (2D) FS cross-section is nearly isotropic. For hole-doped cuprates, the situation is found to be inconclusive, as one FS cross-section is consistent with the tricrystal experiments for random GB tunneling, but not for specular GB tunneling. Another, slightly different hole-doped FS, on the other hand, is consistent with the tricrystal experiments for specular tunneling, but not for random tunneling. The FS of Shirai et al. is inconsistent with most experiments on the hole-doped cuprates, including the tricrystal experiments, for both specular and random GB tunneling. These combined results suggest that defects and small changes in the FS topology play essential roles in interpreting the in-plane GB junction experimental results 1 An important modification to the tetracrystal experiment is also warranted ${ }^{2}$

We let $\theta_{i}$ for $i=L, R$ be the angles the $x_{i}$ axes on the left $(\mathrm{L})$ and right $(\mathrm{R})$ sides make with the normal to a straight GB interface, Fig. 1. GL treatments of the Josephson current $I$ for a $d_{x^{2}-y^{2}}$-wave OP yielded for 


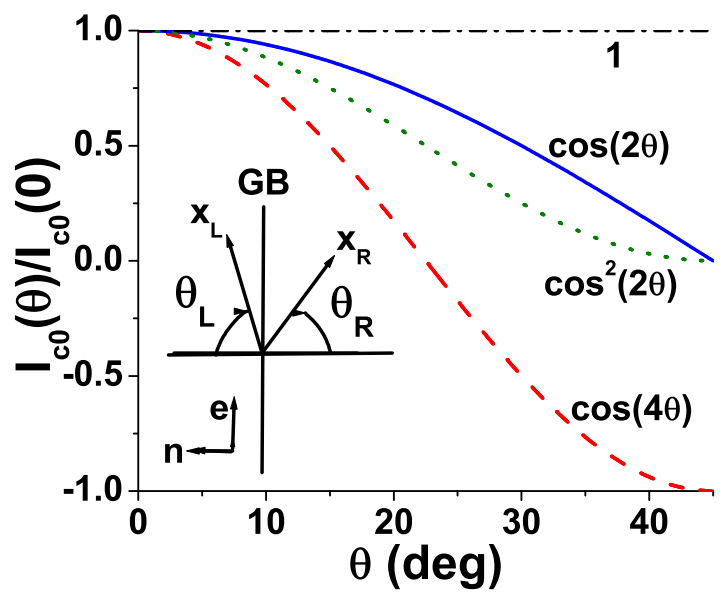

FIG. 1: (Color online) Plots of $I_{c 0}(\theta) / I_{c 0}(0)$ for a $d_{x^{2}-y^{2}}$-wave OP with straight (dotted green), symmetric (dotted green) and asymmetric (solid blue) GB junctions in the ideal GL model, and straight (dot-dashed black), symmetric (dashed red) and asymmetric (solid blue) GB junctions in the faceted GL model. Inset: Sketch of a (001) tilt GB junction.

ideal (non-reconstructed) and faceted GB's,, .22

$$
\begin{aligned}
I_{d}^{i} & =I_{c d}^{i} \cos \left(2 \theta_{L}\right) \cos \left(2 \theta_{R}\right) \sin (\delta \phi), \\
I_{d}^{f} & =I_{c d}^{i} \cos \left[2\left(\theta_{L}+\theta_{R}\right)\right] \sin (\delta \phi) / 2,
\end{aligned}
$$

where $\delta \phi=\phi_{L}-\phi_{R}$ is the OP phase difference across the GB. These have the form $I=I_{c 0} \sin (\delta \phi)$, where $I_{c}=\left|I_{c 0}\right|$ is the critical current. $I_{c 0}>0$ and $I_{c 0}<0$ are the " 0 junction" and " $\pi$-junction" cases, as $I=I_{c} \sin (\delta \phi+\pi)$ in the latter. There are three types of junctions we shall consider. These are asymmetric, symmetric, and straight junctions. We note that other workers have used the terminology "mirror" and "parallel" for symmetric and straight junctions, respectively ${ }^{28}$ For asymmetric junctions, $\theta_{L}=\theta, \theta_{R}=0, I_{d}^{i, f}(\theta) / I_{d}^{i, f}(0)=\cos (2 \theta)$. For symmetric junctions with $\theta_{L}=\theta_{R}=\theta$ and straight junctions with $\theta_{R}=-\theta_{L}=\theta, I_{d}^{i}(\theta) / I_{d}^{i}(0)=\cos ^{2}(2 \theta)$. For faceted symmetric and straight junctions, $I_{d}^{f}(\theta) / I_{d}^{f}(0)=$ $\cos (4 \theta), 1$, respectively. These ideal and faceted GL results shown in Fig. 1 are in qualitative agreement with our results for electron-doped cuprates with random and specular tunneling, respectively, but differ qualitatively from our microscopic results for hole-doped cuprates.

\section{PROCEDURE}

To calculate $I$ across an in-plane GB, we assume the Fourier transform of the quasiparticle Green function matrix in the bulk of the $i$ th superconductor is ${ }^{30}$

$$
\hat{\mathcal{G}}\left(\boldsymbol{k}^{i}, \omega\right)=-\frac{i \omega+\Delta\left(\boldsymbol{k}^{i}\right) \tau_{1}+\xi\left(\boldsymbol{k}^{i}\right) \tau_{3}}{\omega^{2}+\left|\Delta\left(\boldsymbol{k}^{i}\right)\right|^{2}+\xi^{2}\left(\boldsymbol{k}^{i}\right)}
$$

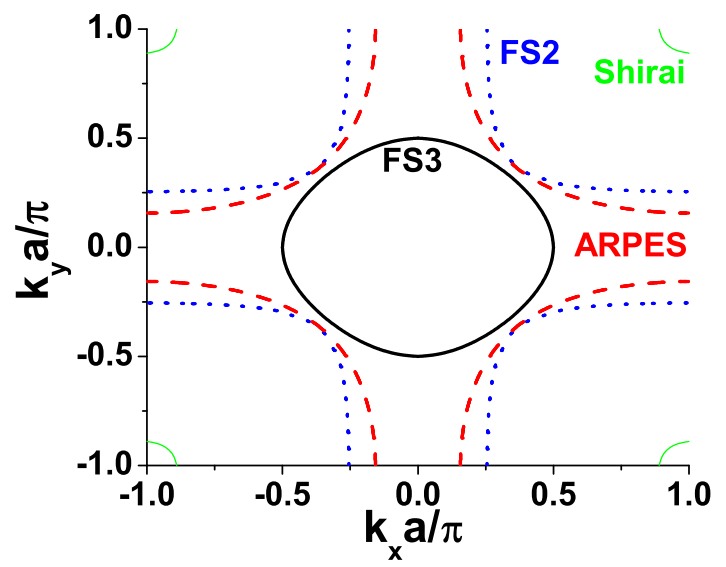

FIG. 2: (Color online) Plots of the four 2D Fermi surfaces studied: The nearly isotropic FS3 (thick solid black) $\stackrel{30}{2}$ the holed-doped FS2 (dotted blue), 30 the best fit to the ARPES FS of Bi2212 (dashed red, ${ }^{29}$ and the extreme hole-doped FS studied by Shirai (thin solid green) ${ }^{28}$

$$
\begin{aligned}
\xi_{0}\left(\boldsymbol{k}^{i}\right)= & -J_{\|}\left[\cos \left(k_{x}^{i} a\right)+\cos \left(k_{y}^{i} a\right)\right. \\
& \left.-\nu \cos \left(k_{x}^{i} a\right) \cos \left(k_{y}^{i} a\right)-\mu\right],
\end{aligned}
$$

where $\omega$ is a Matsubara frequency, $\boldsymbol{k}^{i}$ is the wave vector on the $i$ th GB side, $\Delta\left(\boldsymbol{k}^{i}\right)$ is the respective twodimensional (2D) gap function, $a$ is the tetragonal inplane lattice constant, $\mu, J_{\|}$, and $\nu$ are parameters defining the chemical potential, and in-plane bandwidth and dispersion, respectively, and the $\tau_{j}$ are the Pauli matrices. $\hat{\mathcal{G}}$ is the usual rank 2 matrix with elements $G, F,-G^{\dagger}$, and $F^{\dagger}$ that describes both the single quasiparticle and mean-field pair excitations. We take $\xi\left(\boldsymbol{k}^{i}\right)=$ $\xi_{0}\left(\boldsymbol{k}^{i}\right)+J_{\perp}\left[1-\cos \left(k_{z}^{i} s\right)\right]$, where $s$ is the $c$-axis repeat distance. Here we are mainly interested in the $2 \mathrm{D}$ limit $J_{\perp}=0$. For one tight-binding 2D FS appropriate for Bi2212, FS2, we take $J_{\|}=500 \mathrm{meV}, \mu=0.6$, and $\nu=1.3$, and set $\xi_{0}\left(\boldsymbol{k}_{F}^{i}\right)=0.30$ A slightly different FS, denoted ARPES, that most closely resembles the FS of Bi2212 as observed in ARPES experiments, is obtained with $J_{\|}=306 \mathrm{meV}, \mu=0.675$, and $\nu=0.90 .29$ A nearly circular FS, FS3, is obtained with $\mu=1.0$ and $\nu=0.30$ Finally, we also studied the extreme hole-doped nearneighbor tight-binding FS used by Shirai et al $\stackrel{28}{\approx}$ Taking the maximum value of the gap to be $20 \mathrm{meV}$, this $\mathrm{FS}$ is governed by the parameters $J_{\|}=201 \mathrm{meV}, \nu=0$, and $\mu=-1.94$. These four FS's are pictured in Fig. 2 .

Next, we construct the half-space Green function matrices on the $i$ th side of a straight (001) tilt GB. We let $\hat{\boldsymbol{n}}$ and $\hat{\boldsymbol{e}}$ be unit vectors normal and parallel to the GB satisfying $\hat{\boldsymbol{e}} \times \hat{\boldsymbol{n}}=\hat{\boldsymbol{z}}$, as sketched in Fig. 1. Letting $k_{\|}^{i}=\boldsymbol{k}_{i} \cdot \hat{\mathbf{e}}$ and $k_{\perp}^{i}=\boldsymbol{k}_{i} \cdot \hat{\boldsymbol{n}}$, we set $\mathbf{k}_{\|}^{i}=\left(k_{\|}^{i}, k_{z}^{i}\right)$, $k_{x}^{i}=k_{\perp}^{i} \cos \theta_{i}-k_{\|}^{i} \sin \theta_{i}$, and $k_{y}^{i}=k_{\perp}^{i} \sin \theta_{i}+k_{\|}^{i} \cos \theta_{i}$. The lack of translational invariance requires a discrete indexing of the lattice planes along $\hat{\boldsymbol{n}}$, as in our previous 
technique for $c$-axis tunneling ${ }^{30}$. Thus, for integers $n, m$,

$$
\hat{\mathcal{G}}_{m-n}\left(\boldsymbol{k}_{\|}^{i}, \omega\right)=\int_{-\pi / a_{\perp}^{i}}^{\pi / a_{\perp}^{i}} \frac{d k_{\perp}^{i}}{2 \pi} e^{i k_{\perp}^{i} a_{\perp}^{i}(m-n)} \hat{\mathcal{G}}\left(\boldsymbol{k}^{i}, \omega\right),(5)
$$

where $\boldsymbol{k}_{\|}^{i}=\left(k_{\|}^{i}, k_{z}^{i}\right)$ and the $a_{\perp}^{i} / a=\min \left(\cos \theta_{i}, \sin \theta_{i}\right)$ for $90^{\circ}>\theta_{i}>0^{\circ}, a_{\perp}^{i}=a$ for $\theta_{i}=0^{\circ}, 90^{\circ}$ are the respective lattice plane separations along $\hat{\boldsymbol{n}}$. We choose the GB interface to be between lattice planes 1 and 0 and set $n, m \geq 1, n, m \leq 0$ for $i=R, L$, respectively. Suppressing the $\boldsymbol{k}_{\|}^{i}$ and $\omega$ dependencies, we construct each half-space $\hat{g}_{n m}$ from a combination of the $\hat{\mathcal{G}}_{m n}$ that obeys the free surface boundary conditions (BC's) $\hat{g}_{m 0}=$ $\hat{g}_{0 n}=0$ for $n, m \geq 1$ and $\hat{g}_{m 1}=\hat{g}_{1 n}=0$ for $n, m \leq 0$, obtaining 30.31

$$
\begin{array}{ll}
\hat{g}_{m n}=\hat{\mathcal{G}}_{|m-n|}-\hat{\mathcal{G}}_{m+n}, & \text { for } m, n \geq 1, \\
\hat{g}_{m n}=\hat{\mathcal{G}}_{|m-n|}-\hat{\mathcal{G}}_{2-m-n}, & \text { for } m, n \leq 0 .
\end{array}
$$

We then paste together the two half-space layered superconductors. The supercurrent $I$ across the GB between the two half-spaces is given for a general junction hopping matrix element $J_{J}\left(\boldsymbol{k}_{\| \mid}^{L}, \boldsymbol{k}_{\|}^{U}\right)$ by

$$
\begin{aligned}
I= & \frac{i e T}{2} \sum_{\boldsymbol{k}_{\| \mid}^{L}, \boldsymbol{k}_{\|}^{U}, k_{z}, \omega} \operatorname{Tr}\left[\left(\tau_{0}+\tau_{3}\right)\right. \\
& \times\left(J_{J}\left(\boldsymbol{k}_{\| \mid}^{L}, \boldsymbol{k}_{\|}^{U}\right) \hat{G}_{10}\left(\boldsymbol{k}_{\|}^{U}, \boldsymbol{k}_{\| \mid}^{L}, k_{z}, \omega\right)\right. \\
& \left.\left.-J_{J}\left(\boldsymbol{k}_{\|}^{U}, \boldsymbol{k}_{\| \mid}^{L}\right) \hat{G}_{01}\left(\boldsymbol{k}_{\| \mid}^{L}, \boldsymbol{k}_{\|}^{U}, k_{z}, \omega\right)\right)\right],
\end{aligned}
$$

where $\hat{G}_{n m}\left(\boldsymbol{k}_{\|}^{L}, \boldsymbol{k}_{\|}^{U}, k_{z}, \omega\right)$ is the full Green's function matrix for the combined two half spaces coupled via tunneling across the (001) tilt GB ${ }^{30}$ An example of the resulting interface is pictured in Fig. 3. In principle, it is straightforward to evaluate $I$ to all orders in $J_{J}$, as was done for the case of coherent tunneling across a $c$-axis twist junction ${ }^{30}$ As in that case, however, the most relevant case, especially for GB misalignment angles $\theta_{R}+\theta_{L}$ sufficiently large, is for weak tunneling across the GB $?^{2}$

We set $\Delta\left(\mathbf{k}^{i}\right)=\exp \left(i \phi_{i}\right) \operatorname{Re} \Delta\left(\mathbf{k}^{i}\right)$ and define $\bar{g}_{n m}^{p q}$ to be the $(p q)$ th matrix element of the rank 2 matrix $\hat{g}_{n m}$ with $\phi_{i}=0$. To leading order in the general tunneling matrix element $J_{J}\left(\mathbf{k}_{\| \mid}^{L}, \mathbf{k}_{\| \mid}^{R}\right)$, we obtain

$$
\begin{gathered}
I=\frac{4 e T}{A^{2}} \sum_{\mathbf{k}_{\| \mid}^{R}, \mathbf{k}_{\|,}^{L}, \omega}\left|J_{J}\left(\mathbf{k}_{\|}^{L}, \mathbf{k}_{\| \mid}^{R}\right)\right|^{2} \bar{g}_{11}^{12}\left(\mathbf{k}_{\| \mid}^{R}, \omega\right) \\
\times \bar{g}_{00}^{21}\left(\mathbf{k}_{\|}^{L}, \omega\right) \sin (\delta \phi),
\end{gathered}
$$

where $A$ is the junction area, $e$ is the electron charge, $\delta \phi=\phi_{L}-\phi_{R},-\pi / s \leq k_{z}^{i} \leq \pi / s,-\pi / a_{\|}^{i} \leq k_{\|}^{i} \leq \pi / a_{\|}^{i}$, and the $a_{\|}^{i}=a^{2} / a_{\perp}^{i}$ are the lattice constants along $\hat{\boldsymbol{e}}$. We note that $\bar{g}_{11}^{12}$ and $\bar{g}_{00}^{21}$ are the $F$ and $F^{\dagger}$ pair functions for real OP's on opposite sides of the junction, respectively.

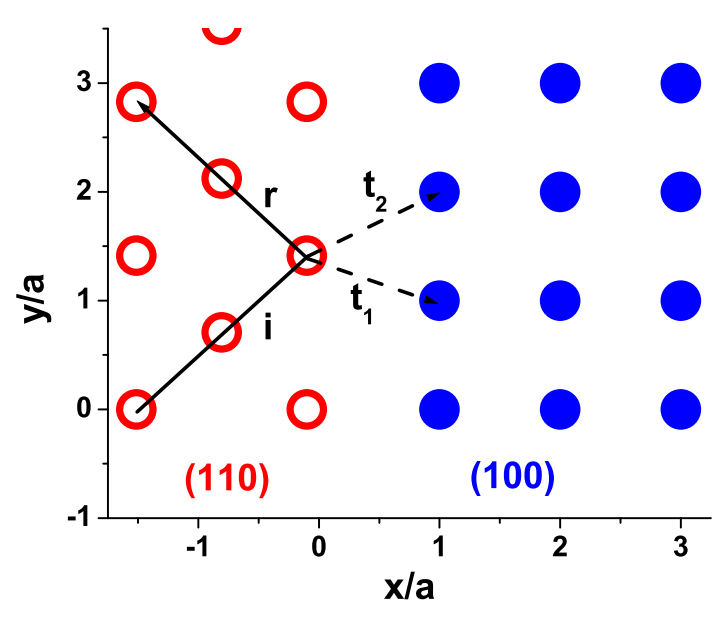

FIG. 3: (Color online) Sketch of a small part of a (110)|(100) junction. The bottom rows of the left (110) lattice (open red circles) and right (100) (filled blue circles) lattice are aligned. An incident quasiparticle can hop along the path $i$, and be specularly reflected along the path $r$, but tunnels non-specularly into the (100) lattice via the two primary initial paths $t_{1}$ and $t_{2}$, as indicated.

We note that although Eq. (9) is proportional to $\sin \delta \phi$ since it is obtained in the limit of weak GB tunneling, our exact expression for the supercurrent, Eq. (8), also contains the full temperature dependence of all of the harmonics proportional to $\sin (n \delta \phi)$ for $n \geq 1$.

Experimentally for large symmetric tilt angles $\theta>$ $\theta_{c} \approx 5-7^{\circ}, I_{c}(\theta) \ll I_{c}(0), 2,32,33$ justifying our weak tunneling assumption, Eq. (9), although the approximation is less accurate for $\theta<\theta_{c}$. Others working on (001) tilt grain boundary junctions of HTSC have also made this assumption, ${ }^{25.34}$ although they used a circular FS and did not necessarily take account of the surface boundary conditions ${ }^{34}$ Since the effects induced by the GB to the real-space $d_{x^{2}-y^{2}}$-wave pairing interaction are formidable, here we only treat the surface $\mathrm{BC}$ effects, and discuss the effects of self-consistency elsewhere ${ }^{35}$ We note that a self-consistent calculation by Tanaka and Kashiwaya for an OP of mixed symmetry suggested no qualitative changes upon the imposition of self-consistency ${ }^{36}$ We include the limiting cases of specular (or coherent) tunneling, where $\boldsymbol{k}_{\|}^{R}=\boldsymbol{k}_{\| \mid}^{L}$, and random (or incoherent) tunneling, where $\boldsymbol{k}_{\|}^{R}$ and $\boldsymbol{k}_{\|}^{L}$ are independent, writing 37

$$
\left|J_{J}\left(\boldsymbol{k}_{\| \mid}^{L}, \boldsymbol{k}_{\|}^{R}\right)\right|^{2}=\left|J_{J}^{\mathrm{sp}}\right|^{2} \delta_{\boldsymbol{k}_{\|}^{R}, \boldsymbol{k}_{\|}^{L}} A+\left|J_{J}^{\mathrm{r}}\right|^{2} .
$$

We remark that the specular (coherent) and random (incoherent) tunneling processes are microscopic processes that take place on an atomic scale. For specular tunneling to occur, the component of the wave vector of the quasiparticle parallel to the junction does not change at each atomic site upon tunneling. With random tun- 
neling, the components of the quasiparticle wave vectors parallel to the junction on the two sides of the junction are completely independent variables. For example, let us consider a tight-binding model of near-neighbor hopping in a tetragonal lattice, as in Eq. (4) with $\nu=0$. In this model, a quasiparticle far from the junction hops from one site to an adjacent site. In a defect-free straight (or symmetric) (100)|(100) junction, the lattices on both sides of the junction are identical. Thus, a quasiparticle can propagate from any site on one side of the junction to the nearest neighbor site on the other side without changing its direction. A quasiparticle can also backscatter coherently at the interface, preserving the component of the wave vector parallel to the junction, as in other treatments $\stackrel{23.25}{=}$ For instance, when the interface is a (110) surface, the surface is theoretically atomically flat, allowing a substantial amount of coherent reflection, as sketched in Fig. 3. Although with the usual vapor phase epitaxy, such (100)|(110) junctions are far from perfect,,$\stackrel{2}{ }$ when they were constructed using the far superior liquid phase epitaxy, an excellent fit to the Fraunhofer pattern was obtained by application of a magnetic field parallel to the junction, without any evidence of a zero-bias conductance peak at the interface,, 38 at least in some cases. Such behavior is consistent with the incoherent tunneling model of Ambegaokar and Baratoff (AB) for an $s$-wave superconductor. In earlier vapor deposition of the somewhat different (110)|(001) GB's, sometimes a rough approximation to the Fraunhofer pattern was observed, with a peak at low fields, and sometimes a very different pattern with a dip at zero field, possibly consistent with a $d$-wave OP $\stackrel{34}{=}$ was observed $\stackrel{40}{*}$ In both of these experiments, high resolution transmission electron microscopy (HRTEM) demonstrated the quality of the junctions 38.40

However, at interfaces such as those containing a (130) surface, Fig. 4, the amount of coherent reflection off the surface is likely to depend strongly upon the incident quasiparticle wave vector direction, as the surface is a periodic, but uneven zigzag. To date, such junctions have never been prepared with the atomic precision of the above two cases 2.4

On the other hand, for an asymmetric (100)|(110) junction, Fig. 3, the ratio of the parallel lattice spacings on the two sides of the junction is $\sqrt{2}$, an irrational number. Although specular reflection at the interface by a quasiparticle is entirely possible, in this case, even if the junction were atomically perfect, the proportion of atomic sites at which coherent tunneling across the junction can occur is vanishingly small. That is, in tunneling to the nearest site across the junction, nearly all quasiparticles will change the component of their direction parallel to the junction. One example of this is sketched in Fig. 3. When one includes the dominant junction tunneling from all sites along the junction, the tunneling component of the quasiparticle wave vector parallel to the junction is effectively random.

In general, one expects that atomically perfect straight

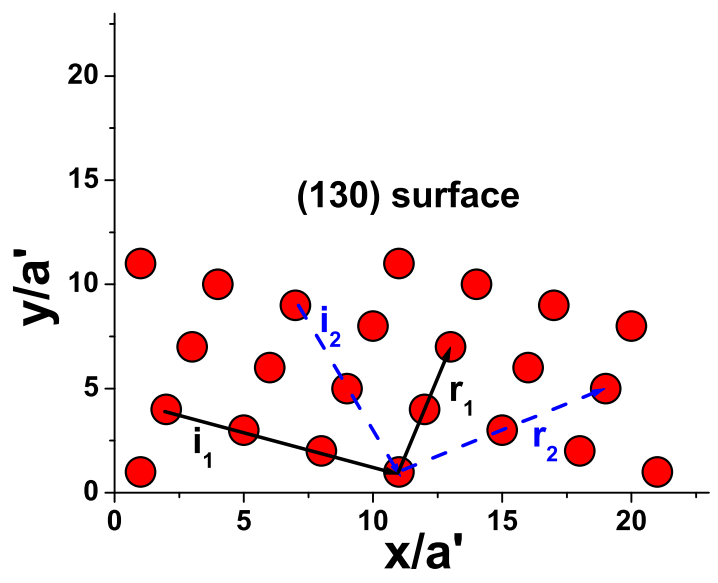

FIG. 4: (Color online) Sketch of the atomic sites (filled red circles) of a tetragonal plane near a (130) surface (the $x$ axis), where $a^{\prime}=\sqrt{10} a$. The solid black and dashed blue arrows indicate selected incident and reflected quasiparticle paths involving only near-neighbor and next-nearest neighbor hopping, respectively.

junctions can exhibit specular, or coherent tunneling. Defects would lead to a component of the tunneling that is random, or incoherent. Asymmetric junctions and symmetric junctions that cannot also be described as straight junctions (such as the (100)|(100) junction) intrinsically lead to predominantly random, or incoherent tunneling. Thus, atomically perfect $(p q 0) \mid(100)$ asymmetric or $(p q 0) \mid(p q 0)$ symmetric junctions for $p+q \neq 1$ (with $\theta_{L}=\theta_{R}$ ) are expected to exhibit primarily random, or incoherent microscopic tunneling processes. Facets, atomic disorder, stoichiometry variations, etc., at the junction contribute further to the randomness of the tunneling processes $32,33,41.42,43,44,45,46$

This microscopic (atomic scale) coherence or incoherence of the tunneling process is completely unrelated to the macroscopic coherence of the superconducting wave function, which can occur over the entire area of the junction, provided that it is prepared sufficiently uniformly. For example, the $\mathrm{AB}$ microscopic model of Josephson tunneling, 39 which averages independently over the parallel components of the quasiparticle wave vector on each side of the junction, assumes uniformly random, or incoherent, microscopic quasiparticle tunneling only. However, that model leads to macroscopic coherence of the superconducting wave function on each side of the junction. Note that although most experiments are consistent with Bi2212 having strongly incoherent intrinsic caxis tunneling between the double layers, the product of the critical current and the normal state resistance $I_{c} R_{n}$ is about $20 \%$ of that expected for a pure $s$-wave superconductor with perfect interfaces, the $\mathrm{AB}$ value ${ }^{4.39}$ This occurs both for intrinsic and for $c$-axis twist junctions several $\mu \mathrm{m}$ in cross-section. $4.16,19,21$ 


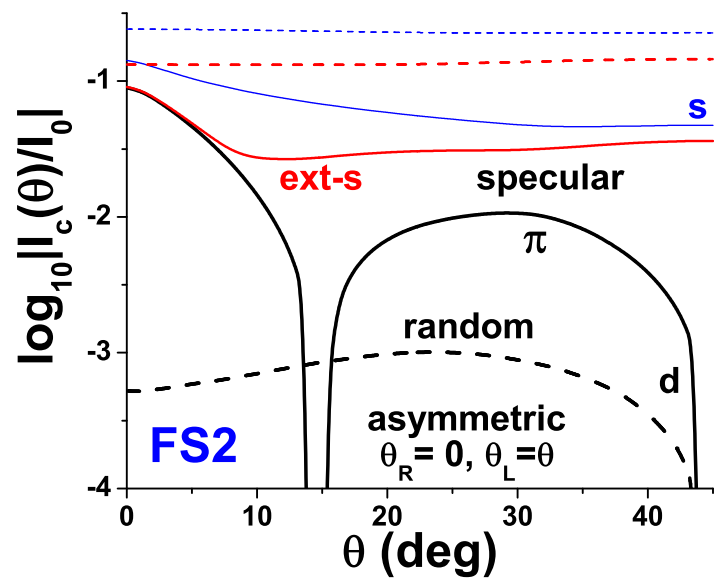

FIG. 5: (Color online) Plots of $\log _{10}\left|I_{c}(\theta) / I_{0}\right|$ for asymmetric junctions with $\theta_{R}=0, \theta_{L}=\theta$, for $s$ - (thin blue), extended- $s$ (intermediate thickness red), and $d_{x^{2}-y^{2}}$-wave (thick black) superconductors, for specular (solid) and random (dashed) GB tunneling and the tight-binding hole-doped FS2. The $d$-wave curves satisfy $I_{c 0}\left(90^{\circ}-\theta\right)=-I_{c 0}(\theta)$, but the $s$ - and extended-s-wave curves satisfy $I_{c 0}\left(90^{\circ}-\theta\right)=I_{c 0}(\theta)$. See text.

\section{RESULTS}

In Fig. 5, we present our results obtained from Eq. (9) for the low- $T I_{c}$ across asymmetric junctions, $\theta_{R}=0, \theta_{L}=\theta$, including the tight-binding FS2 and the surface BC effects. In all of our calculations, we assume the $d_{x^{2}-y^{2}}$ and extended-s-wave OP's have the bulk real-space pairing forms $\Delta_{0}(T)\left[\cos \left(k_{x}^{i} a\right)-\cos \left(k_{y}^{i} a\right)\right]$ and $\Delta_{0}(T) \mid \cos \left(k_{x}^{i} a\right)-$ $\cos \left(k_{y}^{i} a\right) \mid$, respectively. The ordinary-s-wave OP is $\Delta_{0}(T)$, independent of $\boldsymbol{k}^{i}$, 30 We take $\Delta_{0}(0)=10 \mathrm{meV}$, which implies a maximum $d$-wave OP of $20 \mathrm{meV}$. In Fig. 4 , we plotted $\log _{10}\left|I_{c}(\theta) / I_{0}\right|$, where $I_{c}=\left|I_{c 0}\right|$ and $I_{0}=4 e\left|J_{J}^{s p}\right|^{2} /\left[a^{3} s\left(0.1 J_{||}\right)^{2}\right], 4 e\left|J_{J}^{r}\right|^{2} /\left[a^{4} s^{2}\left(0.1 J_{||}\right)^{2}\right]$, respectively 37 The solid (dashed) curves are the results for specular (random) tunneling, respectively. The specular $d$-wave GB junction behaves as a $\pi$-junction only for $14.9^{\circ} \leq \theta \leq 45^{\circ}$ and $75.1^{\circ} \leq \theta \leq 90^{\circ}$, qualitatively different from the GL results pictured in Fig. $1 \Omega^{22}$ Note that the $d$-wave $I_{c}$ for random GB tunneling is small but nonvanishing due to the surface BC's. For this and all other cases of random tunneling across GB's between $d_{x^{2}-y^{2}}$ wave superconductors, neglect of the surface BC's would lead necessarily to $I_{c}=0$. In the $2 \mathrm{D}$ limit with random GB tunneling, however, the asymmetric GB results are qualitatively in agreement with the GL results, except for the overall magnitude of $I_{c}$, which is greatly reduced from that obtained for specular GB tunneling.

We also investigated the role of $J_{\perp}$ for the $d$-wave case. For random GB tunneling, as $J_{\perp}$ increases to $40 \mathrm{meV}$, the broad peak in $I_{c 0}(\theta)$ becomes much flatter, and for much

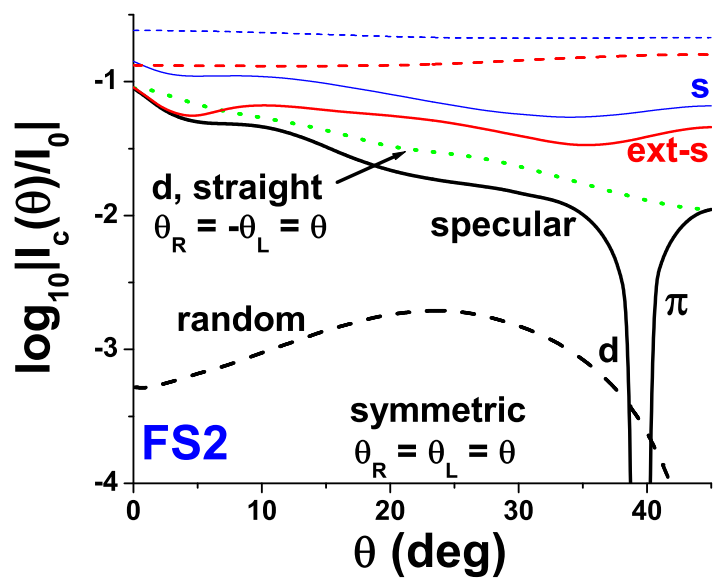

FIG. 6: (Color online) Plots of $\log _{10}\left|I_{c}(\theta) / I_{0}\right|$ for symmetric junctions with $\theta_{R}=\theta_{L}=\theta$, for $s$ - (thin blue), extended- $s$ (intermediate thickness red), and $d_{x^{2}-y^{2}}$-wave (thick black) OP's for specular (solid) and random (dashed) GB tunneling with the tight-binding, hole-doped FS2. Results for straight junctions with $\theta_{R}=-\theta_{L}=\theta$ and a $d_{x^{2}-y^{2}}$-wave OP for specular (dotted green) and random (also dashed black) GB tunneling are also shown. All curves satisfy $I_{c 0}\left(90^{\circ}-\theta\right)=$ $I_{c 0}(\theta)$.

larger $J_{\perp}, I_{c 0}(\theta)$ non-monotonically approaches the GL result $\propto \cos (2 \theta)$. For specular tunneling, $J_{\perp}=10 \mathrm{meV}$ yields $d$-wave results nearly identical to those pictured for $J_{\perp}=0$. Increasing $J_{\perp}$ beyond $J_{\perp 0} \approx 40 \mathrm{meV}$ eliminates the regions within $0 \leq \theta \leq 45^{\circ}$ of $\pi$-junctions, so that the overall region of $\pi$-junctions is for $45^{\circ} \leq \theta \leq 90^{\circ}$, as for the GL case. Hence, for $J_{\perp} \leq J_{\perp 0}$, the specular, tight-binding asymmetric GB $d$-wave results with FS2 are qualitatively different than the GL ones.

In Fig. 6, we present the analogous results for symmetric junctions with FS2. We also plotted our results for straight GB junctions with a $d_{x^{2}-y^{2}}$-wave OP. For random GB tunneling, the straight GB junction results are identical to the dashed black curve for random GB tunneling with symmetric junctions. For specular GB tunneling, the straight junction results are shown as the dotted green curve. Note that for specular tunneling, straight and symmetric junctions have opposite $I_{c}$ values at $\theta=45^{\circ}$. For symmetric GB junctions, the specular $d$-wave junction behaves as a $\pi$-junction for $39.4^{\circ} \leq \theta \leq 50.6^{\circ}$, but behaves as a 0 -junction otherwise. For random tunneling, the symmetric $d$-wave GB junction behaves similarly to the ideal GL model, Eq. (1), as both have $I_{c}\left(45^{\circ}\right)=0$ but never behave as $\pi$ junctions. However, the $d$-wave GL facet model, Eq. (2), has $I_{d}^{f}\left(45^{\circ}\right)=-I_{d}^{f}\left(0^{\circ}\right)$, which is qualitatively different ${ }^{?}$ We note from Figs. 5, 6 that for small angle $\left(0 \leq \theta \leq 5^{\circ}\right)$ specular GB junctions, the extended-s and $d_{x^{2}-y^{2} \text {-wave }}$ OP's lead to indistinguishable $I_{c}(\theta)$. However, for random GB tunneling, the $d$-wave $I_{c}(\theta)$ is much smaller than 


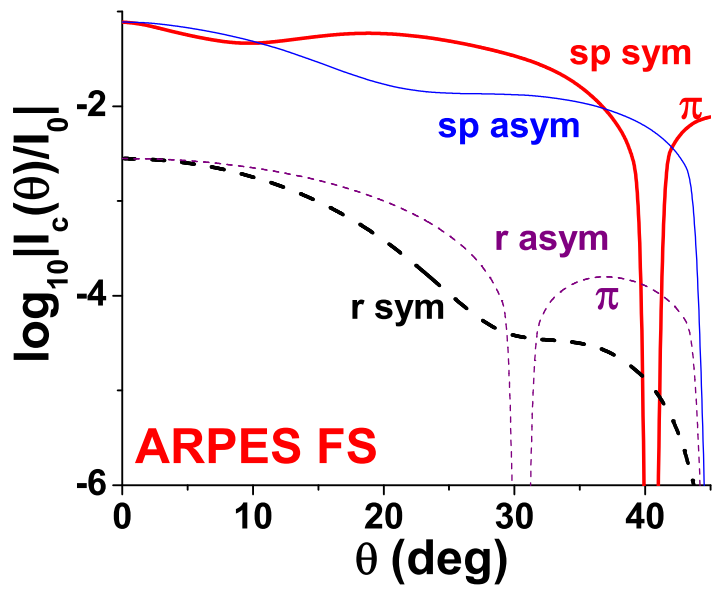

FIG. 7: (Color online) Plots of $\log _{10}\left|I_{c}(\theta) / I_{0}\right|$ for symmetric junctions with specular (thick solid red) and random (thick dashed black) tunneling, and asymmetric junctions with specular (thin solid blue) and random (thin dashed purple) tunneling, for a $d_{x^{2}-y^{2}}$-wave OP with the ARPES FS ${ }^{29}$ The straight and symmetric GB junctions satisfy $I_{c}\left(90^{\circ}-\theta\right)=I_{c}(\theta)$, but the asymmetric GB junctions satisfy $I_{c}\left(90^{\circ}-\theta\right)=-I_{c}(\theta)$.

the extended-s-wave $I_{c}(\theta)$, and would vanish in the absence of the surface BC's. Except for the broad peak and the greatly reduced magnitude of $I_{c}$, the symmetric GB junction across $d_{x^{2}-y^{2}}$-wave superconductors with FS2 and random GB tunneling is qualitatively in agreement with the GL result of Fig. 1 $\stackrel{22}{i}$ For specular GB tunneling, however, our microscopic $d_{x^{2}-y^{2}}$ results differ qualitatively from the GL forms $\underline{22}$

We remark that the ordinary-s- and extended-s-wave OP's lead to $I_{c}(0)$ values that are comparable to each other for both GB tunneling mechanisms. However, for the $d_{x^{2}-y^{2}}$-wave OP, random GB tunneling with FS2 leads to a suppression of $I_{c}(0)$ by a factor of 461 from that of the ordinary-s-wave OP. This suggests that $I_{c}(0)$ for the $d_{x^{2}-y^{2}}$-wave OP with weak, random GB tunneling is unlikely to be consistent with the large values observed experimentally across low-angle (001) tilt junctions ${ }^{2}$ The broad peak in $I_{c 0}(\theta)$ for the $d$-wave OP with random GB tunneling for both asymmetric and symmetric junctions is also inconsistent with most experimental data. On the other hand, the $d$-wave OP with specular GB tunneling across asymmetric junctions does show a rapid decrease in $I_{c 0}(\theta) / I_{c 0}(0)$ with increasing $\theta$, in agreement with lowangle experiments,$\stackrel{2}{=}$ but vanishes at $14.9^{\circ}$ and $39.4^{\circ}$ for FS2, respectively, unlike the experiments ${ }^{2}$

In Figs. 7-9, we compare our $\log _{10}\left|I_{c}(\theta) / I_{0}\right|$ results for $d_{x^{2}-y^{2}}$-wave superconductors across symmetric and asymmetric GB junctions with three different FS's. For each FS, $I_{c}$ results for straight and symmetric GB junctions with random GB tunneling are identical, and for specular GB tunneling, the $I_{c}\left(45^{\circ}\right)$ 's are opposite in sign and equal in magnitude. In Figs. 7-9, the results for sym- metric GB junctions with specular (random) GB tunneling are shown as the thick solid red (dashed black) curves, respectively, and results for asymmetric GB junctions with specular (random) GB tunneling are shown as the thin solid blue (dashed purple) curves, respectively. Results for straight and symmetric GB junctions with random tunneling are identical, and the results for straight GB junctions with specular GB tunneling are shown as the dotted green curves.

In Fig. 7 we plotted $\log _{10}\left|I_{c}(\theta) / I_{0}\right|$ versus $\theta$ for symmetric and asymmetric GB junctions for a $d_{x^{2}-y^{2}}$-wave superconductor with the ARPES FS. Note that for symmetric junctions, a $\pi$-junction occurs with specular GB tunneling for $40.3^{\circ} \leq \theta \leq 49.7^{\circ}$, very similar to the region obtained for FS2 pictured in Fig. 5. In addition, for random GB tunneling across symmetric junctions, no $\pi$-junctions are obtained. However, for asymmetric junctions, the results differ substantially from those obtained for a $d_{x^{2}-y^{2}}$-wave superconductor with FS2, pictured in Fig. 4. In that (FS2) case, the asymmetric GB junction was a $\pi$-junction for specular GB tunneling with $14.9^{\circ}<\theta<45^{\circ}$ and $75.1^{\circ}<\theta \leq 90^{\circ}$, but was a 0 junction otherwise. For random GB tunneling, it was always a 0 -junction for $0 \leq \theta \leq 45^{\circ}$ and a $\pi$-junction for $45^{\circ} \leq \theta \leq 90^{\circ}$. For this slightly different (see Fig. 2) ARPES FS, however, nearly the opposite behavior was found. Asymmetric GB junctions with the ARPES FS with specular GB tunneling are 0 -junctions for $0 \leq \theta<$ $45^{\circ}$ and $\pi$-junctions for $45^{\circ}<\theta \leq 90^{\circ}$, but for random tunneling, a $\pi$-junction appears for $30.6^{\circ}<\theta<45^{\circ}$ (and also for $59.4^{\circ}<\theta \leq 90^{\circ}$ ), and a 0 -junction otherwise. Hence, these slightly different hole-doped FS's lead to qualitatively different $I_{c}$ behavior for $d_{x^{2}-y^{2}}$-wave superconductors. For $s$ - and extended-s-wave superconductors, on the other hand, such strong FS dependence of $I_{c}(\theta)$ does not occur. Although for clarity in presenting the $d$-wave results, we have not shown our $s$ - and extended-s-wave data for the ARPES FS, our calculations confirm the close similarity of those results to the analogous ones pictured in Figs. 5 and 6, confirming the reliability of our results.

With the ARPES FS, asymmetric GB junctions with specular tunneling and for symmetric GB junctions with random tunneling are qualitatively consistent with the ideal GL model ${ }^{22}$ and symmetric GB junctions with specular tunneling are qualitatively consistent with the GL facet model,$\frac{7}{,}$ except that the position of $I_{c}(\theta)=0$ has shifted from $22.5^{\circ}$ to $40.3^{\circ}$. However, asymmetric GB junctions with random tunneling and the ARPES FS are completely unlike either GL model.

Next, we calculated $I_{c 0}(\theta)$ at low $T$ across GB's with the nearly circular FS3, appropriate for the electrondoped cuprates such as NCCO, and presented our results in Fig. 8. For asymmetric GB's, our $d$-wave results agree with the GL model, $I_{c 0}(\theta) / I_{c 0}(0) \approx \cos (2 \theta)$, for both specular and random GB tunneling, but for the latter, $I_{c 0}(0)$ is greatly reduced in magnitude for a $d$ wave OP. Our results for symmetric or straight GB's 


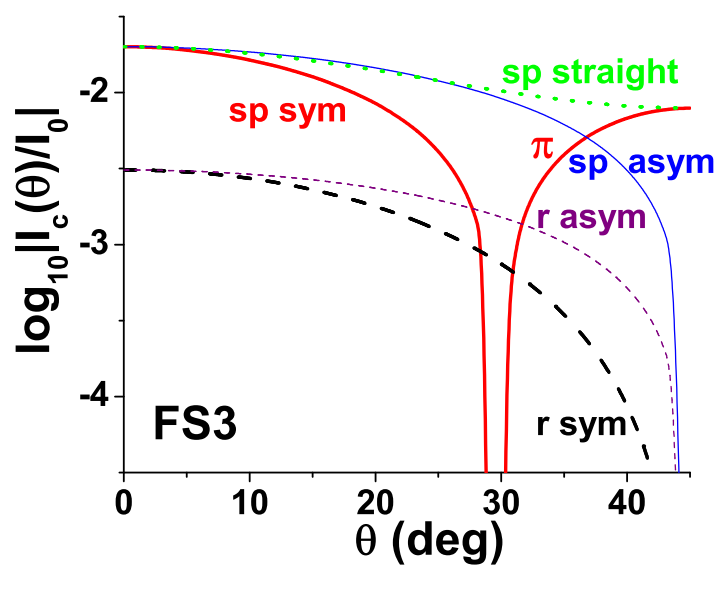

FIG. 8: (Color online) Plots of $\log _{10}\left|I_{c}(\theta) / I_{0}\right|$ for symmetric junctions with specular (thick solid red) and random (thick dashed black) tunneling, and asymmetric junctions with specular (thin solid blue) and random (thin dashed purple) tunneling, for a $d_{x^{2}-y^{2}}$-wave OP with the nearly isotropic, "electrondoped" FS3. Also shown is the specular $d$-wave curve (dotted green) for straight junctions. The straight and symmetric junctions satisfy $I_{c}\left(90^{\circ}-\theta\right)=I_{c}(\theta)$, but the asymmetric junctions satisfy $I_{c}\left(90^{\circ}-\theta\right)=-I_{c}(\theta)$.

with random GB tunneling coincide with each other, and $I_{c 0}(\theta) / I_{c 0}(0) \approx \cos ^{2}(2 \theta)$, as in the GL model for ideal symmetric GB junctions (Fig. 1). For specular GB tunneling across symmetric GB junctions, the results are qualitatively similar to the GL facet model, Eq. (2) except that the $\theta$ value for the crossover from 0 - to $\pi$-junction behavior has shifted from $22.5^{\circ}$ to $29.8^{\circ}$. With that modification, all of our $d$-wave results for the electron-doped FS3 are qualitatively in agreement with the GL facet model for specular GB tunneling, ${ }^{7}$ and with the GL ideal model for random GB tunneling between $d_{x^{2}-y^{2}}$-wave superconductors ${ }^{22}$ This conclusion is somewhat unexpected, as one naïvely might have expected the specular and random results with FS3 to agree with the ideal and facet GL models, respectively. Our low- $T$ symmetric junction results are also qualitatively in agreement with those obtained at low $T$ for weak tunneling with a perfectly circular FS and a FS-restricted $d$-wave OP with different GB roughness $\underset{25}{25}$ and our straight GB junction results with random GB tunneling are qualitatively consistent with previous straight, dirty- $N$, SNS junction results. ${ }^{24}$

In order to compare the results obtained using our technique of imposing the surface BC's upon the Green function matrix to that obtained using different techniques by others, we performed explicit calculations for these purposes. According to the formulas in their paper, Shirai et al. used the FS pictured by the small green pockets near the corners of the first BZ pictured in Fig. $2 \stackrel{28}{\underline{2}}$ Those authors constructed half-spaces exhibiting (100), (130), (120), and (110) surfaces, and pasted them together with specular tunneling in either the straight or symmetric configurations (which they denoted as parallel and mirror, respectively). Their discrete quantization axis was always along the $x$ direction parallel to the $\mathrm{CuO}$ bonds, and hence only normal to the surface for the (100) case. The number of rows in the $y$ direction varied from 1 for the (100) and (110) surfaces to 3 for the (130) surface. Unlike our quantization axis that was defined to be parallel and normal to the surface as pictured in Figs. 3 and 4, upon Fourier transformation in the $y$ direction, their non-normal quantization lattice basis set could lead to unintended hopping across the GB with the strong, intrinsic tight-binding near-neighbor hopping matrix element.

In addition, Shirai et al. included tunneling from a few rows other than the surface row of idealized atomic sites, by assuming that the specular GB tunneling matrix elements decreased inversely instead of exponentially with the tunneling distance, taking the maximum hopping strength to be $0.05 t$, or $6.2 \%$ of the effective near-neighbor hopping strength, $0.803 t \stackrel{28}{\underline{2}}$ Nevertheless, they calculated the single quasiparticle densities of states and $I_{c}$ across the straight and symmetric GB junctions with the above form of specular tunneling, for $\theta=0^{\circ}, 18.4^{\circ}, 26.5^{\circ}$, and $45^{\circ}$, respectively. Some of their low- $T$ limits of $I_{c}$ can be compared with ours using their unusual FS 28 Although they did not mention it explicitly, Shirai et al. found the straight $\theta=0^{\circ}$ and $26.5^{\circ}$, junctions to be 0 -junctions. However, they found strong zero-bias conductance peaks at all junction sites for the straight $45^{\circ}$ junction, and at two of the three distinct junction sites for their $18.4^{\circ}$ junction, suggesting that both of these junctions behave as $\pi$-junctions. For the symmetric junctions, they found the low- $T$ limits of the symmetric $18.4^{\circ}$ and $45^{\circ}$ junctions to be $\pi$-junctions, but the low- $T$ limits of the $0^{\circ}$ and $26.5^{\circ}$ symmetric GB junctions to be 0 -junctions ${ }^{28}$ Of these, $I_{c}(T)$ for the $18.4^{\circ}$ symmetric junction changed sign at a finite $T$ value.

Our low- $T$ results for $\log _{10}\left|I_{c}(\theta) / I_{0}\right|$ versus $\theta$ for symmetric, asymmetric, and straight junctions with the Shirai FS are pictured in Fig. 9. In all cases, even for $\theta=0$, the extremely small overlap of the Fermi surfaces for specular GB tunneling leads to an extremely small $I_{c}$. Our results for specular GB tunneling with symmetric junctions are shown as the thick solid red curves with three alternating regions of $\pi$ - and 0 -junctions for $0 \leq \theta \leq 45^{\circ}$. In the domain $0^{\circ} \leq \theta \leq 45^{\circ}, \pi$-junctions are found in the three regions $1.57^{\circ}<\theta<7.19^{\circ}$, $9.85^{\circ}<\theta<20.89^{\circ}$, and $31.52^{\circ}<\theta<45^{\circ}$. The behavior of the domain $45^{\circ} \leq \theta \leq 90^{\circ}$ is obtained from $I_{c}\left(90^{\circ}-\theta\right)=I_{c}(\theta)$. Random tunneling across either straight or symmetric GB junctions leads to just a 0junction for $0 \leq \theta \leq 90^{\circ}$, although there are strong dips in $I_{c}$ at $\theta \approx 13.6^{\circ}$ and $76.4^{\circ}$ ) (not pictured) and $I_{c}\left(45^{\circ}\right)=0$, as indicated by the thick dashed black curve. For asymmetric GB junctions with specular GB tunneling, the thin solid blue curve shows a $\pi$-junction for $17.04^{\circ}<\theta<45^{\circ}$ (and from $72.96^{\circ}<\theta \leq 90^{\circ}$ ), 


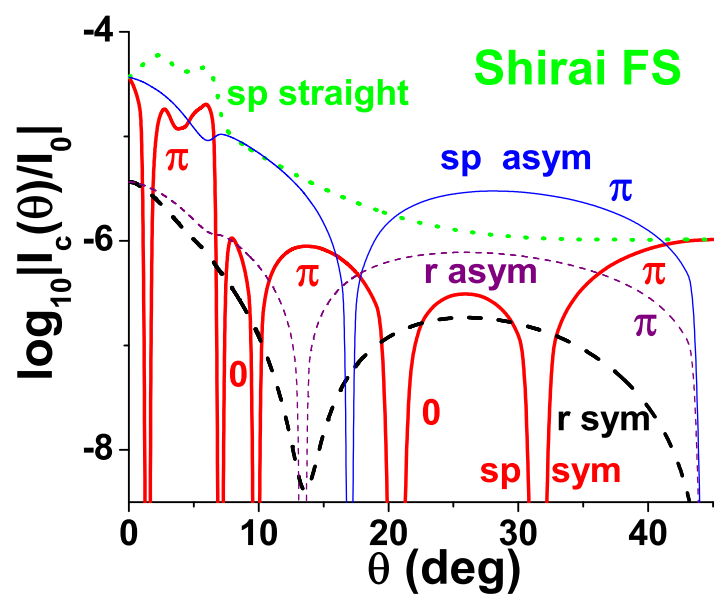

FIG. 9: (Color online) Plots of $\log _{10}\left|I_{c}(\theta) / I_{0}\right|$ for symmetric junctions with specular (thick solid red) and random (thick dashed black) tunneling, and asymmetric junctions with specular (thin solid blue) and random (thin dashed purple) tunneling, for a $d_{x^{2}-y^{2}}$-wave OP with the extreme hole-doped FS of Shirai et al. ${ }^{28}$ Also shown is the specular $d$-wave curve (dotted green) for straight junctions. The straight and symmetric junctions satisfy $I_{c}\left(90^{\circ}-\theta\right)=I_{c}(\theta)$, but the asymmetric junctions satisfy $I_{c}\left(90^{\circ}-\theta\right)=-I_{c}(\theta)$. The thick dashed black curve represents 0 -junctions only.

and the thin dashed purple curve for random GB tunneling indicates a $\pi$-junction for $13.34^{\circ}<\theta<45^{\circ}$ (and from $76.66<\theta \leq 90^{\circ}$ ). In addition, the dotted green curve represents straight junctions with specular tunneling, which are always 0 -junctions, as in the ideal and faceted GL models pictured in Fig. 1, and in all other FS's we studied (e. g., Figs. 6 and 8).

Some of our results and those of Shirai can be directly compared. Both procedures lead to $T=0 \pi$-junctions for symmetric GB junctions with specular GB tunneling with $\theta=18.4^{\circ}, 45^{\circ}$, but 0 -junctions at $\theta=0^{\circ}, 26.5^{\circ}$. As for straight junctions, our results for all four FS's we studied always lead to 0-junctions, as for both the ideal and faceted GL models. For straight junctions at the two angles $0^{\circ}$ and $26.5^{\circ}$, Shirai et al. also obtained 0junctions for all $T \leq T_{c}$. However, as mentioned above, Shirai et al. appear to have obtained $\pi$-junctions for the straight junctions with $\theta=18.4^{\circ}$ and $45^{\circ}$. In particular, we find their apparent $\pi$-junctions for the two straight junctions, $\theta=18.4^{\circ}, 45^{\circ}$ to be difficult to understand. Suppose Shirai et al. were to increase their dominant junction coupling strength from $0.05 t$ to their bulk hopping strength, $0.803 t$. Then, the junction would be invisible to the quasiparticles, and one would certainly not expect any surface effects, such as in the straight $0^{\circ}$ junction they studied ${ }^{28}$ Although Shirai et al. attributed their peculiar results to the oddness of the number of site rows in their minicell, we suspect that their results may be artifacts of their peculiar minicell basis, which was not

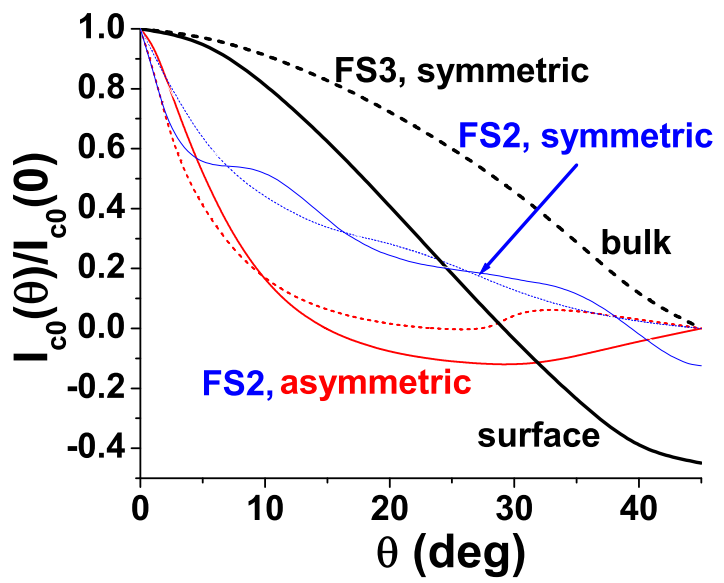

FIG. 10: (Color online) Plots of $I_{c 0}(\theta) / I_{c 0}(0)$ for the $d_{x^{2}-y^{2-}}$ wave OP with specular tunneling, with (solid) and without (dashed) the surface boundary conditions, for symmetric GB junctions with the nearly circular FS3 (thick black) and the tight-binding FS2 (thin red) and, and for asymmetric GB junctions with FS2 (intermediate thickness blue). The symmetric curves satisfy $I_{c}\left(90^{\circ}-\theta\right)=I_{c}(\theta)$, and the antisymmetric curves satisfy $I_{c}\left(90^{\circ}-\theta\right)=-I_{c}(\theta)$.

defined to be parallel and perpendicular to the GB, as in Fig. 4.28

Finally, in Fig. 10 we compare our specular $I_{c 0}(\theta) / I_{c 0}(0) d$-wave results with the surface BC's with those obtained from the bulk Green functions. We compare those results obtained with the tight-binding FS2 for asymmetric (Fig. 5) and symmetric (Fig. 6) junctions, and also our results for symmetric junctions obtained with the nearly circular FS3 (Fig. 8). For both symmetric GB cases, there are no regions in $0^{\circ} \leq \theta \leq 45^{\circ}$ of $\pi$-junctions for the bulk calculations. Including the surface effects, these symmetric GB cases have $\pi$-junctions for $39.4^{\circ}<\theta<50.6^{\circ}$ and $29.2^{\circ}<\theta<55.8^{\circ}$ for FS2 and FS3, respectively. For asymmetric junctions with FS2, the bulk calculation leads to weak $\pi$-junctions for $23.7^{\circ}<\theta<28.3^{\circ}$ with a negative slope at $45^{\circ}$, yielding additional $\pi$-junctions for $45^{\circ}<\theta<61.7^{\circ}$ and $66.3^{\circ}<\theta \leq 90^{\circ}$. The surface BC's greatly enhance the magnitude and range of such $\pi$-junctions to $14.9^{\circ}<\theta<45^{\circ}$ and $75.1^{\circ}<\theta \leq 90^{\circ}$, and change the sign of the slope at $45^{\circ}$. Although not pictured in Fig. 10 , the surface BC's play an essential role in the case of random tunneling across GB's between $d$-wave superconductors. In the absence of the surface BC's, $I_{c}=0$ in such cases. Thus, we conclude that the surface BC effects cannot be ignored, especially with regard to $d_{x^{2}-y^{2}}$-wave superconductors. 


\section{DISCUSSION}

Tricrystal experiments were performed for a variety of cuprate superconductors 1.7 .8 .9 Tricrystal (a) consists of one asymmetric $0^{\circ} / 60^{\circ}$ junction and two $0^{\circ} / 30^{\circ}$ junctions. Tricrystal (b) consists of two $0^{\circ} / 71.6^{\circ}$ junctions and one symmetric $18.4^{\circ} / 18.4^{\circ}$ junction. Tricrystal (c) consists of two asymmetric $0^{\circ} / 73^{\circ}$ junctions and an asymmetric $2^{\circ} / 32^{\circ}$ junction ${ }^{1.10}$ It was claimed that an odd number of $\pi$-junctions was observed only for tricrystal (a) ${ }^{1.10}$ In the case of Bi2212, only results for tricrystal (a) were shown 8.9

First of all, our results for the electron-doped FS3 with either specular or random GB tunneling are in complete agreement with a predominant $d_{x^{2}-y^{2}}$-wave OP as determined from the tricrystal experiments on $\mathrm{NCCO} 10$ That is, one would only expect an odd number of $\pi$-junctions only for tricrystal (a), for both random and specular GB tunneling.

However, for hole-doped cuprates, the agreement with the tricrystal experiments is less robust. Assuming only a $d_{x^{2}-y^{2}}$-wave OP, 7 our FS2 results pictured in Figs. 3, 4 show that tricrystal (a) would have two $\pi$-junctions if the tunneling were specular, and one $\pi$-junction if it were random. Tricrystal (b) should have no $\pi$-junctions, as claimed. Tricrystal (c), if the $2^{\circ} / 32^{\circ}$ junction can be approximated by an asymmetric $0^{\circ} / 34^{\circ}$ junction, would have one $\pi$-junction for specular tunneling, and two $\pi$ junctions for random tunneling. Hence, our FS2 results are only consistent with the observation claims if the tunneling is non-specular.

On the other hand, the ARPES FS, while only slightly different from FS2, leads to very different conclusions with regard to the agreement with the tricrystal experiments. For specular GB tunneling, the ARPES FS is in complete agreement with the observations of the three tricrystal experiments. However, for random GB tunneling, tricrystal (a) is expected to have a very small overall $I_{c}$, as $I_{c} \approx 0$ for all three asymmetric GB junctions in that tricrystal. Although tricrystal (b) would be expected to exhibit an even number of $\pi$-junctions, as claimed to be observed, tricrystal (c) would be expected to exhibit one $\pi$-junction for random GB tunneling with the ARPES FS. Hence, the ARPES FS can only be fully consistent with the tricrystal experiments on hole-doped cuprates for non-random GB tunneling.

One interpretation is that if the quasiparticle tunneling across the relevant grain boundaries is random and the OP were pure $d$-wave, then the FS would be expected to be more like FS2. In the unlikely event that the grain boundary tunneling were coherent, then a pure $d$-wave OP would have a FS more like the ARPES FS. Such a scenario might be used to argue that the FS of Bi2212, usually thought to have the ARPES FS form, is actually closer to FS2. However, we caution that FS2 and the ARPES FS are one-band approximations to the FS of Bi2212, which contains two $\mathrm{CuO}_{2}$ layers thought by many to be responsible for the superconductivity, and that no tricrystal (c) experiments were performed on Bi2212. In addition, this scenario ignores the $c$-axis twist experiments, which indicate that essentially all of the $c$-axis tunneling is in the $s$-wave channel $\stackrel{4}{*}$ If those three experiments are correct, then Bi2212 contains an OP with an $s$-wave component that is at least $20 \%$ of the total. Although not appreciated by Tsuei et al. $\frac{9}{,}$ in the presence of nanoscale disorder that has recently been observed in STM experiments,, 33 a mixed $d+s$ OP is indeed allowed 4 Thus, it is possible that Bi2212 could have a dominant $d$-wave OP and still have all of the intrinsic $c$-axis tunneling be in the $s$-wave OP channel. If this scenario proves to be true, then further calculations with a mixed $s$ - and $d$-wave OP would be warranted. ${ }^{20}$

With regard to the most-studied material, YBCO, in addition to the two $\mathrm{CuO}_{2}$ layers, there is also a conducting $\mathrm{CuO}$ chain layer, which is responsible for the orthorhombicity and the strong twinning that occurs in thin films. The strong twinning makes (100) and (010) films indistinguishable, which can complicate the analysis. Second, unless strongly underdoped, YBCO appears to be more three-dimensional than are most other cuprates, so that $J_{\perp} \neq 0$ might be large enough to cause an important modification to our results. Third, it is clear from many other experiments that YBCO also has a substantial $s$-wave component $, 13,14$ which has often been ignored by many workers $\frac{1,9}{1}$ In each twin domain, a purported dominant $d$-wave OP would have either a $d+s$ or a $d-s$ OP mix, and if the twin areas were equal, then one might expect the $s$-wave OP component to make a negligible contribution to the measured critical currents. However, in $c$-axis $\mathrm{YBCO} / \mathrm{Pb}$ Josephson junctions made with highly twinned YBCO thin films, quantitative agreement to the Fraunhofer pattern was obtained, although $I_{c} R_{n}$ was considerably less than the AB value ${ }^{15}$

Thus, the different results of FS2 and the ARPES FS indicate that the tricrystal experiments are less robust determinations of the symmetry of the order parameter in hole-doped cuprates than has been generally thought. Small variations in the FS topology and the non-specularity of the GB tunneling processes play a crucial role that cannot be anticipated a priori. To the extent that defects play a role in the tunneling processes, it might be possible to understand the experimental observations with a substantial $s$-wave OP component. Especially since the (001) tilt GB junctions are known to be increasingly underdoped with increasing GB misalignment,, 2.32 .33 .42 it is possible that $\pi$-junctions arising from the local magnetism of the $\mathrm{Cu}^{+}$spins formed from oxygen loss at the GB junctions could be important.

All of our calculations using the Shirai FS are in complete disagreement with both the tricrystal and tetracrystal experiments, regardless of the degree of randomness in the GB tunneling matrix elements. In addition, the Shirai et al. results for specular symmetric $18.4^{\circ} / 18.4^{\circ} \mathrm{GB}$ 's are in direct conflict with all experiments using tricrystal (b) 1,28 Taking all of our results for the various FS's into account, it appears that there is a topological crossover 
relevant for the agreement of the critical currents with the GL results, ${ }^{22}$ with full agreement for the electrondoped FS's centered about the $\Gamma$ point in the first BZ, and complete disagreement with the extreme Shirai FS. The cross-over appears to occur for FS's intermediate to the ARPES FS and FS2. Hence, for the hole-doped cuprates, not only are the details of the tunneling important, but the topological structure of the hole-doped FS is also very relevant, and minute details can change the interpretation.

Tetracrystals consisting of highly twinned thin films of the hole-doped YBCO and electron-doped LCCO containing a symmetric $0^{\circ} / 0^{\circ}$ junction and a $45^{\circ} / \pm 45^{\circ}$ junction, plus two symmetric $22.5^{\circ} / 22.5^{\circ}$ junctions were made ${ }^{11,12}$ For comparison, they also made bicrystals with two symmetric $22.5^{\circ} / 22.5^{\circ}$ junctions. We also calculated the straight GB junction case $\theta_{R}=-\theta_{L}=\theta$ for a $d_{x^{2}-y^{2}}$-wave OP, and our results for FS2 and FS3 are shown by the green dotted lines in Figs. 6 and 8, and at $45^{\circ}$, are opposite in sign to the respective symmetric junction cases with specular GB tunneling. If the tunneling were non-specular, as required for the $d$-wave interpretation with FS2 of the tricrystal experiments, then the $45^{\circ} / \pm 45^{\circ}$ junction would have the smallest (or vanishing, for random tunneling) $I_{c}$ value for a $d$ wave OP. If instead the tunneling were specular, as required for the ARPES FS $d$-wave interpretation of the tricrystal experiments on hole-doped cuprates, then that junction would allow a small but significant $I_{c}$ value, but would behave either as a $\pi$ - or 0 -junction, respectively, for a $d$-wave OP, depending upon the particular distribution of twin domains present at the junction. Thus, this tetracrystal would have an indeterminate number of $\pi$-junctions for specular tunneling and a $d$-wave OP, but no supercurrent for random tunneling. Similar arguments apply to the LCCO case. For a highly twinned thin film junction, both specular and random tunneling across the $45^{\circ} / \pm 45^{\circ} \mathrm{GB}$ junction would lead to

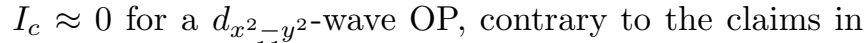
the experiment $11^{1}$ The other two junctions, symmetric $22.5^{\circ} / 22.5^{\circ}$ junctions, would not be $\pi$-junctions, unless the tunneling were specular and the FS were different from any that we studied. Hence, the interpretation put forward by those authors, which assumed the $45^{\circ} / \pm 45^{\circ}$ junctions were straight, should be reexamined, ${ }^{11,12}$ as the $\pi$-junction could arise extrinsically from defects in this very imperfect $45^{\circ} / \pm 45^{\circ}$ junction ${ }^{2.32 .33 .42}$ We urge that the experiment be redesigned in the form of a tricrystal, omitting the $45^{\circ} / \pm 45^{\circ}$ junction.

Recently, there have been zigzag in-plane $\mathrm{YBCO} / \mathrm{Nb}$ experiments ${ }^{47.48}$ In these experiments, a YBCO film is angularly cut from above with a ramping angle $\varphi \approx$ $15-20^{\circ}$ into a regular zigzag pattern of nominally exposed (100) and (010) edges, each edge of equal length $\ell \approx 10-40 \mu \mathrm{m}$. After deposition of a thick layer of $\mathrm{Au}$, a thicker layer of $\mathrm{Nb}$ was applied. This experiment is a variation of the earlier $\theta=90^{\circ} \mathrm{YBCO} / \mathrm{Pb}$ superconducting quantum interference device (SQUID) experiment of
Mathai et al. ${ }^{49}$ which contained $\mathrm{YBCO} / \mathrm{Pb}$ junctions with a thin $\mathrm{Ag}$ interstitial layer along the ramped nominally (100) and (010) edges of YBCO, respectively. In those experiments, one of the junctions functioned as a 0 -junction, and the other functioned as a $\pi$-junction 49 The beauty of the recent experiment is that the zigzag array of alternating $0-/ \pi$-junctions was observed by a SQUID microscope to exhibit an ordered array of fractional vortices, the magnitude of which was within the experimental error of $\Phi_{0} / 2$, where $\Phi_{0}$ is the flux quantum. Although no microscopic analysis of the edges of the YBCO was presented, the ion milling technique employed necessarily leaves a rough surface on a scale of at least 1-5 nm, so that the tunneling across each of these edges is most likely incoherent. The thick Au layer could modify the overall tunneling process, however. The much longer length scales $\lambda_{J}$ of the Josephson vortices, which is only 1-2 orders of magnitude smaller than the edge dimensions, allows the vortices to weakly interact, forming a regular array. These experiments are suggestive of a dominant $d$-wave OP.

However, we recall that Gim et al. studied $\mathrm{YBCO} / \mathrm{Pb}$ SQUID's prepared with a much wider selection of SQUID angles $\theta{ }^{50}$ In those experiments, which did not include $\theta=90^{\circ}$, the results were inconsistent with either a predominant $d$ - or $s$-wave OP component,, 50 but were instead consistent with a $p$-wave polar state. Apparently, the preparation of a 0 -junction or a $\pi$-junction depends upon the ramping angle $\varphi$ of the bombarding ions, so that many pairs of SQUID's with angles $\theta$ and $\theta+180^{\circ}$ contained one 0 -junction and one $\pi$-junction 50 Although the nice zigzag experiments do demonstrate the reproducibility of the 0 -junction and $\pi$-junction preparation at fixed $\varphi$ and $\theta=90^{\circ}$ with $\mathrm{YBCO} / \mathrm{Nb}$ junctions, those authors did not report the results of $\theta$ and $\varphi$ variation studies, 47.48

We are presently engaged in calculating the single quasiparticle and pair excitations at low $T$, both of which are contained in the Green function matrix given by Eqs. (6) and (7). For brevity we have here only presented the Josephson critical current across the grain boundaries, the quasiparticle densities of states at the sites on the rows adjacent to or near to the junction can be evaluated from the imaginary part of the single particle $g_{n n}^{11}$ for $n=0,1, \ldots$ Although the most commonly used procedure for obtaining the zero-bias conductance peak on a surface is to use the Bogoliubov-deGennes technique, ${ }^{23}$ our Green function technique with the appropriate sur-

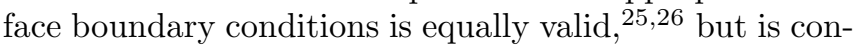
siderably more compact and powerful. With our Green function technique, we are able to evaluate the critical current at arbitrary grain boundary angles, and are not restricted to specific interfaces such as $(p q 0) \mid\left(p^{\prime} q^{\prime} 0\right)$ for integral $p, q, p^{\prime}, q^{\prime}$. It is also much easier to generalize the tunneling forms, although we have limited our treatment to the most commonly studied specular and random cases. Although it would be rather easy to generalize the tunneling matrix elements to forms intermediate to these 
limiting cases for symmetric and straight junctions, $\stackrel{29}{2}$ for asymmetric junctions, such a generalization is more difficult. We have compared our results with those obtained by Shirai et al. using a purely numerical technique, and some of their numerical results at low $T$ are in qualitative agreement with our results obtained from Eq. (9). Our results with the nearly circular FS3 are in fairly good agreement with those obtained with the GL model and with microscopic calculations using a circular FS.7.22.25 The main drawback with the Green function technique is the procedure for making the calculations self-consistent. However, as noted by Tanaka and Kashiwaya, ${ }^{36}$ making the results self-consistent does not usually result in qualitative changes. We are presently engaged in selfconsistent calculations for various grain boundaries of the $(p q 0) \mid\left(p^{\prime} q^{\prime} 0\right)$ types, as was done by Shirai et al..$^{28}$ but we are using the near-neighbor and next-nearest neighbor single quasiparticle matrix elements appropriate for either the ARPES or FS2 FS's on Bi2212, and will present our results elsewhere ${ }^{35}$ We will also present the quasiparticle densities of states for these more realistic FS models elsewhere 35

Finally, we remark that recent YBCO thin film structures have been studied using (001)|(103) thin film boundaries with $45^{\circ}$ in-plane misalignment angles, and the results interpreted using the Sigrist-Rice formula 57.58 We have not yet analyzed such grain boundaries, because the Fermi surface of YBCO is particularly complicated, and our FS2 and ARPES FS's are likely to be inadequate to do so reliably. Nevertheless, from the results we obtained with these two FS's, it is evident that the results obtained from such devices, the grain boundaries of which have not been analyzed with atomic scale precision as was done with the Bi2212 $c$-axis twist experiments, ${ }^{4}$ might also have a more conventional explanation. $51,52,53,54,55,56$

It has been suggested that the experimental $I_{c}(\theta)$ observed in (001) tilt grain boundary junctions can only be understood in terms of interface defects, regardless of the OP. ${ }^{42}$ Of course, in Bi2212, it has long been established that a significant amount of static defects exist not only on the top cleaved surface,, 43 but throughout even the best single crystals. ${ }^{44,45,46}$ Such defects combined with significant oxygen loss at the grain boundaries may be the most important source of the observed $\pi$-junctions in the tricrystal and tetracrystal experiments, accounting for the apparent inconsistency between those and the $c$-axis bicrystal and cross-whisker experiments, 4.18 .19 .21

Finally, we recall that $\pi$-junctions are now routinely produced using junctions of the SNS and SINIS varieties constructed from conventional superconductors, $, 51,52,53,54,55,56$ so that a close reexamination of the tri- and tetracrystal experiments is warranted. This is especially true in the case of the hole-doped cuprates. We note that not only are $\pi$-junctions readily obtained with $\mathrm{Nb}, \mathrm{Al}$ and their oxides, 51 but the associated zero-bias conductance peaks and higher harmonic order Josephson phase relations in such conventional junctions have also been observed, $51,52,53,54,55,56$ as in $\mathrm{YBCO}, 57,58$

\section{SUMMARY}

We calculated the critical current $I_{c}$ versus (001) inplane tilt angle $\theta$ for symmetric and asymmetric grain boundary junctions, assuming the order parameter has either the $s^{-}$, extended-s-, or $d_{x^{2}-y^{2}}$-wave form. We used two Fermi surfaces appropriate for hole-doped cuprates, one appropriate for electron-doped cuprates, and an extreme Fermi surface for comparison purposes, and took account of the surface boundary conditions appropriate for the interfaces. We studied both tunneling limits of specular and random tunneling. An important feature of our results is that the presence of the surface boundary conditions allows for a finite $d$-wave critical current across junctions in which the microscopic tunneling is random. This is especially important because the tunneling across nearly all in-plane (001) tilt junctions is expected to be random on an atomic scale, even within each reconstructed facet.

Although our results for electron-doped cuprates are in complete agreement with the interpretation of the observation of the tricrystal experiments on those compounds, our results for hole-doped cuprates differ qualitatively with those obtained from Ginzburg-Landau models, and indicate that the interpretation of the tricrystal experiments is complicated, depending upon strong assumptions about the details of the Fermi surface topology and the nature of the tunneling processes. In addition, corresponding problems in the interpretation of the tetracrystal experiments on hole-doped cuprates persist, leading to expectations of an $I_{c}(\theta)$ inconsistent with many experiments. 2 We urge further in-plane (001) tilt grain boundary experiments using films deposited by liquid phase epitaxy to be made, and for high resolution transmission electron microscopy studies of the pertinent grain boundaries to be presented. ${ }^{2.4 .38}$

\section{Acknowledgments}

The authors would like to thank T. Claeson, S. E. Shafranjuk, and A. Yurgens for useful discussions.

\footnotetext{
* Electronic address: garnold@nd.edu
}

$\dagger$ Electronic address: klemm@phys.ksu.edu 
1 C. C. Tsuei and J. R. Kirtley, Rev. Mod. Phys. 72, 969 (2000).

2 H. Hilgenkamp and J. Mannhart, Rev. Mod. Phys. 74, 485 (2002).

3 K. A. Müller, Phil. Mag. Lett. 82, 279 (2002).

4 R. A. Klemm, Phil. Mag. 85, 801 (2005); Int. J. Mod. Phys. B 12, 2920 (1998).

${ }^{5}$ R. A. Klemm in Nonequilibrium Physics at Short Times Scales. Formation of Correlations, edited by K. Morawetz (Springer, Berlin, 2004), pp. 381-400.

6 P. Chaudhari and S. Y. Lin, Phys. Rev. Lett. 72, 1084 (1994).

7 C. C. Tsuei, J. R. Kirtley, C. C. Chi, L. S. Yu-Jahnes, A. Gupta, T. Shaw, J. Z. Sun, and M. B. Ketchen, Phys. Rev. Lett. 73, 593 (1994).

8 J. R. Kirtley, C. C. Tsuei, H. Raffy, Z. Z. Li, A. Gupta, J. Z. Sun, and S. Megtert, Europhys. Lett. 36, 707 (1996).

9 C. C. Tsuei, J. R. Kirtley, G. Hammer, J. Mannhart, H. Raffy, and Z. Z. Li, Phys. Rev. Lett. 93, 187004 (2004).

10 C. C. Tsuei and J. R. Kirtley, Phys. Rev. Lett. 85, 182 (2000).

11 R. R. Schulz, B. Chesca, B. Goetz, C. W. Schneider, A. Schmall, H. Bielefeldt, H. Hilgenkamp, J. Mannhart, and C. C. Tsuei, Appl. Phys. Lett. 76, 912 (2000).

12 B. Chesca, K. Ehrhardt, M. Mößle, R. Straub, D. Koelle, R. Kleiner, and A. Tsukada, Phys. Rev. Lett. 90, 057004 (2003).

13 A. G. Sun, D. A. Gajewski, M. B. Maple, and R. C. Dynes, Phys. Rev. Lett. 72, 2267 (1994); A. G. Sun, A. Truscott, A. S. Katz, R. C. Dynes, B. W. Veal, and C. Gu, Phys. Rev. B 54, 6734 (1996).

14 R. Kleiner, A. S. Katz, A. G. Sun, R. Summer, D. A. Gajewski, S. H. Han, S. I. Woods, E. Dantsker, B. Chen, K. Char, M. B. Maple, R. C. Dynes, and J. Clarke, Phys. Rev. Lett. 76, 2161 (1996).

15 A. S. Katz, A. G. Sun, R. C. Dynes, and K. Char, Appl. Phys. Lett. 66, 105 (1995).

16 M. Mößle and R. Kleiner, Phys. Rev. B 59, 4486 (1999).

17 S. I. Woods, A. S. Katz, T. L. Kirk, M. C. de Andrade, M. B. Maple, and R. C. Dynes, IEEE Trans. Appl. Supercond. 9, 3917 (1999).

18 Qiang Li, Y. N. Tsay, M. Suenaga, R. A. Klemm, G. D. $\mathrm{Gu}$, and N. Koshizuka, Phys. Rev. Lett. 83, 4160 (1999).

19 Y. Takano, T. Hatano, S. Kawakami, M. Ohmi, S. Ikeda, M. Nagao, K. Inomata, K. S. Yun, A. Ishii, A. Tanaka, T. Yamashita, and M. Tachiki, Physica C 408-410, 296 (2004).

20 R. A. Klemm, Phys. Rev. B 67, 174509 (2003).

21 Yu. I. Latyshev, A. P. Orlov, A. M. Nikitina, P. Monceau, and R. A. Klemm, Phys. Rev. B 70, 094517 (2004).

${ }^{22}$ M. Sigrist and T. M. Rice, J. Phys. Soc. Jpn. 61, 4283 (1992).

23 Y. Tanaka and S. Kashiwaya, Phys. Rev. Lett. 74, 3451 (1995); Phys. Rev. B 53, R11957 (1996); ibid. 56, 892 (1997).

24 Y. Asano, Phys. Rev. B 64, 014511 (2001); Y. Asano, Y. Tanaka, and S. Kashiwaya, Phys. Rev. B 69, 134501 (2004).

25 Yu. S. Barash, H. Burkhardt, and D. Rainer, Phys. Rev. Lett. 77, 4070 (1996).

26 M. S. Kalenkov, M. Fogelström, and Yu. S. Barash, Phys. Rev. B 70, 184505 (2004).

27 E. Il'ichev, M. Grajcar, R. Hlubina, R. P. J. IJsselsteijn, H. E. Hoenig, H.-G. Meyer, A. Golubov, M. H. S. Amin, A. M.
Zagoskin, A. N. Omelyanchouk, and M. Yu. Kupriyanov, Phys. Rev. Lett. 86, 5369 (2001).

28 S. Shirai, H. Tsuchiura, Y. Asano, Y. Tanaka, J. Inoue, Y. Tanuma, and S. Kashiwaya, J. Phys. Soc. Jpn. 72, 2299 (2003). In their Eq. (15), these authors defined their quasiparticle dispersion to be $\epsilon_{\boldsymbol{q}}=-\left(t+\zeta_{1}\right) \eta_{\boldsymbol{q}}-\zeta_{2} \gamma_{\boldsymbol{q}}-$ $(\mu+8 W n)$, with $\eta_{\boldsymbol{q}}=2\left(\cos q_{x}+\cos q_{y}\right)$, their Eq. (17), in units of the lattice constant $a=1$. Following their Eq. (21), the definitions $t=W, \zeta_{1}=-0.19 t, \zeta_{2}=0.0 t$, $\mu=-0.2833 t$, and the hole density $n=\left\langle\sum_{\sigma} n_{\boldsymbol{r}, \sigma}\right\rangle=0.85$ were given, where the summation is only over the two spin states. Putting these numbers into the above dispersion, one obtains $\epsilon_{\mathbf{q}}=-1.606 t\left(\cos q_{x}+\cos q_{y}+4.06\right)$, which never vanishes, and hence does not exhibit a Fermi surface. However, the $\boldsymbol{q}$-independent part of this expression is inconsistent with their Eq. (4) for the effective chemical potential, $\tilde{\mu}=\mu+W \sum_{\rho, \sigma}\left\langle n_{\boldsymbol{\rho}, \sigma}\right\rangle$, where the summation is over the four near-neighbor in-plane sites denoted by $\rho$ and the two spin states. Hence, the 8 in their Eq. (15) should be replaced with the number 4 to avoid overcounting the two spin states. With this modification, the correct dispersion of Shirai et al. becomes $\epsilon_{\mathbf{q}}=-1.606 t\left(\cos q_{x}+\cos q_{y}+1.94\right)$, which has the Fermi surface at small pockets in the corners of the first Brillouin zone shown in Fig. 2. Although Shirai et al. may have intended to include this factor of 4 in their definition of $n$, which would have led to a Fermi surface resembling FS2 in Fig. 2, from the equations in their manuscript that they very likely used the above dispersion corresponding in our notation to $J_{\|}=201 \mathrm{meV}$, $\nu=0$, and $\mu=-1.94$, where $J_{\|}$can be determined from their value of $\Delta=0.0799 t$, which we set equal to $10 \mathrm{meV}$. However, their strange results for the symmetric (mirror) and especially the straight (parallel) $18.4^{\circ}$ junctions do not appear to correspond to any results by other authors for any FS studied.

29 A. Bille, R. A. Klemm, and K. Scharnberg, Phys. Rev. B 64, 174507 (2001).

30 G. B. Arnold and R. A. Klemm, Phys. Rev. B 62, 661 (2000).

31 S. H. Liu and R. A. Klemm, Phys. Rev. Lett. 73, 1019 (1994).

32 X. Y. Cai, A. Gurevich, I.-F. Tsu, D. L. Kaiser, S. E. Babcock, and D. C. Larbalestier, Phys. Rev. B 57, 10951 (1998).

33 M. F. Chisholm and S. J. Pennycook, Nature (London) 351, 47 (1991).

34 X.-Z. Yan and C.-R. Hu, Phys. Rev. Lett. 83, 1656 (1999).

35 G. B. Arnold and R. A. Klemm, unpublished.

36 Y. Tanaka and S. Kashiwaya, Phys. Rev. B 58, R2948 (1998).

37 R. A. Klemm, G. Arnold, C. T. Rieck, and K. Scharnberg, Phys. Rev. B 58, 14203 (1998).

${ }^{38}$ Yu. Eltsev, K. Nakao, Y. Yamada, I. Hirabayashi, Y. Ishimaru, K. Tanabe, Y. Enomoto, J. Wen, and N. Koshizuka, Physica C 367, 24 (2002).

39 V. Ambegaokar and A. Baratoff, Phys. Rev. Lett. 10, 486 (1963); ibid. 11, 104 (1963).

40 Y. Ishimaru, J. Wen, N. Koshizuka, and Y. Enomot, Phys. Rev. B 55, 11851 (1997).

41 X. F. Zhang, D. J. Miller and J. Talvacchio, J. Mater. Res. 11, 2440 (1996).

42 A. Gurevich and E. A. Pashitskii, Phys. Rev. B 57, 13878 (1998) and references therein; ibid. 63, 139901(E) (2001). 
${ }^{43}$ K. M. Lang, V. Madhavan, J. E. Hoffman, E. W. Hudson, H. Eisaki, J. Z. Sun, and S. Megtert, Nature (London) 415 , 412 (2002).

44 Y. Gao, P. Coppens, D. E. Cox, and A. R. Moodenbaugh, Acta Cryst. A 49, 141 (1993); P. Coppens, P. Lee, G. Yang, and H. S. Sheu, J. Phys. Chem. Solids 52, 1267 (1991); V. Petricek, Y. Gao, P. Lee, and P. Coppens, Phys. Rev. B 42, 387 (1990).

45 X. B. Kan and S. C. Moss, Acta Cryst. B 48, 122 (1992).

46 D. Grebille, H. Leligny, A. Ruyter, P. Labbé, and D. Raveau, Acta Cryst. B 52, 628 (1996).

47 H. J. H. Smilde, Ariando, D. H. A. Blank, G. J. Gerritsma, H. Hilgenkamp, and H. Rogalla, Phys. Rev. Lett. 88, 057004 (2002).

${ }^{48}$ H. Hilgenkamp, Ariando, H.-J. H. Smilde, D. H. A. Blank, G. Rijnders, H. Rogalla, J. R. Kirtley, and C. C. Tsuei, Nature (London) 422, 50 (2003).

49 A. Mathai, Y. Gim, R. C. Black, A. Amar, and F. C. Wellstood, Phys. Rev. Lett. 74, 4523 (1995); Anna Mathai, Ph. D. Thesis, University of Maryland, 1995.

${ }^{50}$ Y. Gim, A. Mathai, R. C. Black, A. Amar, and F. C. Wellstood, J. Phys. (Paris) I 6, 2299 (1996); Yonggyu Gim, Ph. D. Thesis, University of Maryland, 1996.

51 J. J. A. Baselmans, A. F. Morpurgo, B. J. van Wees, and
T. M. Klapwijk, Nature (London) 397, 43 (1999); J. J. A. Baselmans, B. J. van Wees, and T. M. Klapwijk, Appl. Phys. Lett. 79, 2940 (2001); J. J. A. Baselmans, T. T. Heikkilä, B. J. van Wees, and T. M. Klapwijk, Phys. Rev. Lett. 89, 207002 (2002).

${ }^{52}$ R. Shaikhaidarov, A. F. Volkov, H. Takayanagi, V. T. Petrashov, and P. Delsing, Phys. Rev. B 62, R14649 (2000).

53 J. Huang, F. Pierre, T. T. Heikkilä, F. K. Wilhelm, and N. O. Birge, Phys. Rev. B 66, 020507(R) (2002).

${ }^{54}$ F. Giazotto, T. T. Heikkilä, F. Taddei, R. Fazio, J. P. Pekola, and F. Beltram, Phys. Rev. Lett. 92, 137001 (2004).

55 I. P. Nevirkovets, S. E. Shafranjuk, and J. B. Ketterson, Phys. Rev. B 68, 024514 (2003).

56 T. M. Klapwijk, J. Supercond.: Incorp. Novel Magn. 17, 593 (2004).

57 T. Lindström, S. A. Charlebois, A. Ya. Tzalenchuk, Z. Ivanov, M. H. S. Amin, and A. M. Zagoskin, Phys. Rev. Lett. 90, 117002 (2003).

58 T. Bauch, F. Lombardi, F. Tafuri, A. Barone, G. Rotoli, P. Delsing, and T. Claeson, Phys. Rev. Lett. 94, 087003 (2005). 